\title{
A note on the projection properties of Hadamard matrices obtained by the first Payley construction
}

\author{
M. Jainz \\ Department of Mathematical Statistics, University of Bern, Sidlerstr. 5, 3012 Bern, Switzerland \\ (E-mail: michael.jainz@stat.unibe.ch)
}

\begin{abstract}
We show that the projections on four factors of an arbitrary orthogonal array of strength 2 allow the estimation of main effects and twofactor interactions when all other effects are assumed to be zero, if those projections satisfy the bounds given by Weil's theorem. The only exceptions are the Hadamard matrices of orders 16 and 24. A consequence is again the estimability of main effects and two-factor interactions for the projections on four factors of the first Payley construction for arbitrary run size.
\end{abstract}

Key words: Hadamard matrix; First Payley construction; Weil's theorem; Projections

\section{Bounds for the first Payley-construction}

Let $H_{n}$, a normalized Hadamard matrix of order $n \geq 12$, be the design matrix of a nonreplicated factorial design, any column except the first corresponding to a factor. For any $k \leq n$ columns let the design matrix $M$ of the "projected design" consist of the first column of $H_{n}$, the $k$ columns of the factors and all columns obtained by Hadamard products of these $k$ columns. We will call this the "complete projected design $M$ in $k$ factors". The corresponding parameter vector $\beta$ consists of the overall mean, the main effects and all interactions of those $k$ factors. Denote the $n \times 1$ observation vector by $Y$. If the matrix $M^{t} M$ has full rank, then the least-squares estimate is

$$
\hat{\beta}=\left(M^{t} M\right)^{-1} M^{t} Y
$$

If there is a priori information that some entries in $\beta$ are zero, we use $\eta$ and $V$ for the parameter and the design matrix of the reduced model. We are mainly concerned with $k=4$ and $\eta$ will consist of all main effects and two-factor interactions. 
We will abuse language and call the sum over the entries of a Hadamard product of an arbitrary number of vectors the "Hadamard product". In the case of two vectors this is just the usual scalar product of two vectors. We will use the notation IK for the Hadamard product of the $i$ 'th and $k^{\prime}$ th column of $M^{t} M$ or $V^{t} V$. In the same way the Hadamard products of more than two columns are denoted.

By definition the off-diagonal entries of $M^{t} M$ or $V^{t} V$ are Hadamard products of factor columns. Because $H_{n}$ is an orthogonal array of strenth 2 , those Hadamard products will consist of three or more columns. In the case of $k=4$ and $\eta, V$ there are 4 different Hadamard products of three and 1 of four columns. Because the first column of $M$ or $V$ is orthogonal to all other columns we will from now on omit this first column. This does not change a zero or nonzero determinant of $M^{t} M$ or $V^{t} V$.

In the following we will examine conditions under which those two matrices have a nonzero determinant.

Consider a finite field with at least three elements, the number of elements of that field being the power of a prime number $p$. We will use the usual abbreviation $G F\left(p^{k}\right), k$ a positive integer. The first Payley-construction of a Hadamard matrix is as follows: For $G F\left(p^{k}\right)=\left\{a_{1}, \ldots, a_{p^{k}-1}, a_{p^{k}}=0\right\}$ define the matrix $A=\left(a_{i j}\right)$ of order $p^{k}+1$ as follows:

$$
\begin{aligned}
& a_{i 0}=a_{0 j}=1 \\
& a_{i j}=\chi\left(a_{i}-a_{j}\right) \quad \text { if } \quad i \neq 0, j \neq 0, i \neq j \\
& a_{i i}=-1
\end{aligned}
$$

Denoting the Hadamard product of the $i^{\prime}$ th, $j^{\prime}$ th and $k^{\prime}$ th rows of A by IKJ, then:

$$
\begin{aligned}
|I K J| & \leq\left|\sum_{a \in G F\left(p^{k}\right)} \chi(a-i) \chi(a-j) \chi(a-k)\right|+4 \\
& =\left|\sum_{a \in G F\left(p^{k}\right)} \chi((a-i)(a-j)(a-k))\right|+4
\end{aligned}
$$

The same holds for the Hadamard product of the $i$ 'th, $j$ 'th, $k^{\prime}$ th and l'th row:

$$
\begin{aligned}
|I K J L| & \leq\left|\sum_{a \in G F\left(p^{k}\right)} \chi(a-i) \chi(a-j) \chi(a-k) \chi(a-l)\right|+5 \\
& =\left|\sum_{a \in G F\left(p^{k}\right)} \chi((a-i)(a-j)(a-k)(a-l))\right|+5
\end{aligned}
$$

Bounds for the sum on the right side of (1) and (2) can be given using Weil's theorem ([3], theorem 5.41.), denoting by $n=p^{k}+1$ the order of the Hadamard matrix:

$$
\begin{aligned}
\left|\frac{I K J}{n}\right| & \leq \frac{\left|\sum_{a \in G F\left(p^{k}\right)} \chi((a-i)(a-j)(a-k))\right|+4}{n} \\
& \leq \frac{2 \sqrt{n-1}+4}{n} \rightarrow 0 \quad(n \rightarrow \infty)
\end{aligned}
$$




$$
\begin{aligned}
\left|\frac{I K J L}{n}\right| & \leq \frac{\left|\sum_{a \in G F\left(p^{k}\right)} \chi((a-i)(a-j)(a-k)(a-l))\right|+5}{n} \\
& \leq \frac{3 \sqrt{n-1}+5}{n} \rightarrow 0 \quad(n \rightarrow \infty)
\end{aligned}
$$

From (3) and (4) follows immediately:

Theorem 1. Let $M$ denote the design matrix of the complete projected design of any $k$ factors of a Hadamard matrix $H_{n}$ obtained by the first Payley construction. Then

$$
\frac{1}{n} M^{t} M \rightarrow I_{2^{k}} \quad(n \rightarrow \infty)
$$

Here $I_{2^{k}}$ is the $\left(2^{k} \times 2^{k}\right)$-unit matrix.

This is, of course, nothing else than the usual asymptotic normality of the least-squares estimate. Nevertheless, in the special case of the first Payley construction, $\frac{1}{n} V^{t} V$ reaches a nonzero determinant quite fast:

\section{The projections on four factors: a computer search}

Suppose now that all interaction effects between three and four factors are zero. Denote the design matrix in this case by $V$.

In the following, we will need some facts from matrix theory and some properties of a (general) orthogonal array of strength 2:

1. A matrix A is said to fulfill the "dominant main-diagonal criterion", if in any row the sum of the absolute values of the off-diagonal entries is strictly smaller than the diagonal entry. In this case $\operatorname{det}(A) \neq 0 \quad$ ([5], chapter 3).

2. For a partitioned matrix

$P=\left(\begin{array}{ll}A & B \\ C & D\end{array}\right)$

where $A$ is an invertible square matrix and $D$ a square matrix, the determinant of $P$ is given by:

$\operatorname{det}(P)=\operatorname{det}(A) \operatorname{det}\left(D-C A^{-1} B\right)$

In the case of the $(10 \times 10)$-matrix $P:=\frac{1}{n} V^{t} V$, the left upper $4 \times 4$ principal minor (a unit matrix) will play the role of $A$, so $\operatorname{det}\left(\frac{1}{n} V^{t} V\right) \neq 0$ if and only if $\operatorname{det}\left(D-C A^{-1} B\right) \neq 0$. The $(6 \times 6)$-matrix $D-C A^{-1} B$ is called the "Schur complement" of the matrix $P$ ([6], Theorem 3.1.1).

3. For any three columns $i, j$ and $k$ of the $(n \times n-1)$-matrix $V$ let $n_{i j k}$ be the number of row vectors $(1,1,1)$. It is not difficult to show that the Hadamard product IJK is then given by:

$I J K=8 n_{i j k}-n$ 
So the entries of $V^{t} V$ are quite restricted $(m \in N)$ :

$$
\begin{aligned}
& I J K \in\{0, \pm 8, \pm 16, \pm 24, \ldots\} \text { if } n=8 m \\
& I J K \in\{ \pm 4, \pm 12, \pm 20, \ldots\} \text { if } n \neq 8 m
\end{aligned}
$$

For any four columns i,j,k and 1 a similar formula holds. Let $n_{i j k l}$ be the number of row vectors $(1,1,1,1)$. Then the Hadamard product IJKL is:

$$
I J K L=3 n-8\left(n_{i j k}+n_{i j l}+n_{i k l}+n_{j k l}\right)+16 n_{i j k l}
$$

Now 1.), 2.) and 3.) make the computer search fast. 1.) and 2.) show that $\operatorname{det}\left(\frac{1}{n} V^{t} V\right) \neq 0$ if $\mathrm{n}$ is already $\geq 68$, using Weil's theorem. We did the computer search only with property 3 .), so:

Theorem 2. Suppose the projections on 3 and 4 factors of an arbitrary $O A$ in $n$ runs $(n \geq 12)$ of strength 2 fulfill the conditions (3) and (4) of Weil's theorem. Then:

All main effects and two-factor interactions of the projected design on any 4 factors can be estimated when all other effects are zero.

The only exceptions: $n=16,24$.

The special case $n=24$ can be eliminated for the first Payley construction using property 3.), here:

$$
\operatorname{det}\left(V^{t} V\right)=0 \Leftrightarrow|I J K L|=|I J K|=|I J L|=|I K L|=|J K L|=8
$$

This is not possible due to the construction of the matrix. We stress the fact that this is the only case where one actually has to examine the structure of a Hadamard matrix. This gives:

Corollary. Suppose $H_{n}$ is a Hadamard matrix obtained by the first Payley construction and $n \geq 12$. Then all main effects and two-factor interactions of the projected design on any 4 factors can be estimated when all other effects are zero.

After this work was completed, the author was informed that Bulutoglu and Cheng ([2]), using another approach (see [1]), obtained a result similar to the given Corollary. Their result is more complete in a sense that they can avoid using a computer and they do not have to make the exception $n=24$.

Acknowledgements. The research was supported in part by the Swiss National Science Foundation. The author is grateful to Prof. Dr. Jürg Hüsler and the Department of Mathematical Statistics, University of Bern, for the given support.

\section{References}

[1] Cheng CS (1995) Some projection properties of orthogonal arrays. Ann. Stat. 23:1223

[2] Bulutoglu DA, Cheng CS. Hidden projection properties of some non-regular fractional factorial designs and their applications. To appear in Ann. Stat. 
[3] Lidl R, Niederreiter H (1983) Finite Fields. Encyclopedia of Mathematics and its Applications, Addison-Wesley

[4] Lin D, Draper NR (1993) Generating alias relationships for two-level Plackett and Burman designs. Comput. Statist. Data Anal. 15:147

[5] Marcus M, Minc H A (1992) Survey of Matrix Theory and Matrix Inequalities. Dover Publications

[6] Prasolov VV. Problems and Theorems in Linear Algebra. Translation of Mathematical Monographs. Vol. 134, The American Mathematical Society

[7] Wang JC, Wu C, Jeff F (1995) A hidden projection property of Plackett-Burman and related designs. Statist. Sinica 5:235 